\title{
Comparative Analysis of HPLC Fingerprint Combined with DAD Detection between Bupleuri Radix and Bupleuri Herba
}

\author{
E Sun, Changmei Wang, Yingjie Wei*, Xiaobin Jia \\ Key Laboratory of New Drug Delivery System of Chinese Meteria Medica, Jiangsu Provincial Academy of Chinese Medicine, Nanjing, China \\ *Corresponding author: wyj970@163.com
}

Received April 02, 2015; Revised April 13, 2015; Accepted April 19, 2015

\begin{abstract}
Bupleuri Herba is widely distributed and traditionally used for the similar clinical application of Bupleuri Radix in Jiangsu and Anhui province in China. To elucidate the difference of material basis between Bupliuri Radix and Bupleuri Herba, a HPLC fingerprint method combined with DAD detection was developed for effective components analysis of these two herb medicines. Both flavonoids and saikosaponins components were analyzed with a zorbax C-18 column by gradient elution using $0.1 \%(\mathrm{v} / \mathrm{v})$ phosphoric acid - acetonitrile as the mobile phase, the column was maintained at $35^{\circ} \mathrm{C}$, the flow rate was $1 \mathrm{~mL} / \mathrm{min}$, the detection wavelength was set at $360 \mathrm{~nm}$ for flavonoids and $203 \mathrm{~nm}$ for saikosaponins. Rutin and saikosaponin d were selected as reference compound, respectively. And correlation analysis was used to calculate the correlation coefficient. The results showed that 18 major flavonoids were detected in both Bupleuri Radix and Bupleuri Herba with only 12 common components peaks; nine major saikosaponins were detected in these two herb medicines with only four common components peaks. Correlation analysis indicated that both Bupleuri Radix and Bupleuri Herb can be divided into two groups according to correlation coefficient difference. In addition, poor correlation was found between Bupleuri Radix and Bupleuri Herb. Comparative analysis of chromatographic fingerprint indicated that the difference of material basis between Bupleuri Radix and Bupleuri Herb was significant, which hinted that clinical application of Bupleuri Herb instead of Bupleuri Radix should be cautious.
\end{abstract}

Keywords: Bupleuri Radix, Bupleuri Herba, HPLC-DAD fingerprint, material basis

Cite This Article: E Sun, Changmei Wang, Yingjie Wei, and Xiaobin Jia, "Comparative Analysis of HPLC Fingerprint Combined with DAD Detection between Bupleuri Radix and Bupleuri Herba.” Journal of Food and Nutrition Research, vol. 3, no. 4 (2015): 267-273. doi: 10.12691/jfnr-3-4-6.

\section{Introduction}

Bupleuri Radix, dry roots belonging to umbelliferae of Bupleurum chinense DC. or Bupleurum scorzonerifolium Willd., is one of the most popular Traditional Chinese Medicines (TCMs) used in China for approximately 2000 years. It has the function of evacuating fever and pyretolysis, soothing liver and relieving depression and lifting the spirit. It has been used to treat cold fevers, chills, chest and hypochondrium gas pains and so on [1]. Bupleuri Herba, the whole plant with roots of Bupleurum scorzonerifolium Willd., is widely distributed and traditionally used for the similar clinical application of Bupleuri Radix in Jiangsu and Anhui province in China. But unlike Bupleuri Radix, Bupleuri Herba has not yet been recorded in Chinese Pharmacopoeia. Whether Bupleuri Herba and Bupleuri Radix have similar function or not? And whether Bupleuri Herba can substitute Bupleuri Radix for clinical use or not? Material basis difference between these two herb medicines may help us to clarify these questions.
It is well known that the major components of Bupleuri Radix and Bupleuri Herba are saikosaponins and flavonoids, respectively. Previous reports about component analysis mainly focused on saikosaponins of Bupleuri Radix [1,2,3,4,5] or flavonoids from Bupleuri Herba [6,7]. Nowadays, researches are beginning to focus on flavonoids in Bupleuri Radix [8,9] or saikosaponins in Bupleuri Herba [10]. However, except our previous quantification on two saikosaponins and four flavonoids of these two herb medicines [11], no other simultaneous analysis on these two major types of components has been reported to date. Chromatographic fingerprint technology is increasingly becoming an effective means for analysis on complex material basis of traditional Chinese medicine $[12,13,14]$. In the present study, HPLC-DAD chromatographic fingerprint was established for simultaneous detection and comparison on both saikosaponins and flavonoids of Bupleuri Radix and Bupleuri Herba, which may enrich analysis methods of Bupleuri Radix and Bupleuri Herba greatly, and may lead us to systematically elucidate material basis difference between them. 


\section{Materials and Methods}

\subsection{Materials and Reagents}

Ten batches of Bupleuri Radix and ten batches of Bupleuri Herba were collected from different provinces of China. All the samples were authenticated by the author as the dried roots of Bupleurum chinense DC. and the dried roots or whole plant of Bupleurum scorzonerifolium Willd. The voucher specimens were deposited in Jiangsu Provincial Academy of Chinese Medicine, Nanjing, PR China. rutin (Lot No. 100080-200707), quercetin (Lot No. 100081-200406), kaempferide (Lot No. 110861-200808), isorhamnetin (Lot No. 110860-200406), saikosaponin a (Lot No. 110777-200406) and saikosaponin d (Lot No. 110778-200505) (purity $\geq 98.0 \%$ ) were purchased from National Institute for the control of Pharmaceutical and Biological Products (Beijing, China). HPLC grade acetonitrile and phosphoric acid were obtained from Merck (Merck, Germany), deionized water was purified by Milli-Q system (Millipore, Bedford, MA, USA), and the other reagents were of analytical grade.

\subsection{Chromatographic Conditions}

The Agilent 1100 HPLC system with G1311A quaternary gradient pump and G1313A autosampler and G1316A column heaters and G1315B DAD detector; Chemstation 6.01 chromatographic work station (Agilent, USA).

Column, Agilent Zorbax Ecllipse plus-C18 (particle size $5 \mu \mathrm{m}$, diameter $4.6 \mathrm{~mm}$, length $250 \mathrm{~mm}$ ); mobile phase, $0.1 \%$ phosphoric acid (A) - acetonitrile (B), the linear gradient elution (0 15min, 5\%B-20\%B; 15 30min, 20\%B-25\%B; 30 60min, 25\%B-45\%B; 60 90min, 45\%B-85\%B; 90 100min, $85 \% \mathrm{~B}-100 \% \mathrm{~B}$ ); flow rate: $1.0 \mathrm{~mL} / \mathrm{min}$; detection wavelength: $203 \mathrm{~nm}$ for saikosaponins and $360 \mathrm{~nm}$ for flavonoids; Column temperature: $35^{\circ} \mathrm{C}$.

\subsection{Preparation of Sample Solutions}

Bupleuri Radix and Bupleuri Herba were grinded into powder (passed through a 65-mesh sieve). Every sample powder (2.0g) was weighed accurately and placed in conical flask with stopper, and added $40 \mathrm{ml}$ of $70 \%$ methanol, then sealed and treated with ultrasound ( power $200 \mathrm{~W}$, frequency $40 \mathrm{kHz}$ ) for $30 \mathrm{~min}$, cooled and weighed again, supplemented the weight loss with $70 \%$ methanol, filtered and the residue washed with $70 \%$ methanol, then the combined filtrate was evaporated to dryness at $50^{\circ} \mathrm{C}$ on a water bath. The residue was dissolved in $70 \%$ methanol and transferred to $10 \mathrm{~mL}$ volumetric flask, diluted to the mark, then shaken and filtered with $0.45 \mu \mathrm{m}$ filter membrane for the test solutions.

\subsection{Preparation of Reference Solutions}

Appropriate quantities of rutin, quercetin, kaempferide and isorhamnetin were weighed accurately and dissolved in methanol, then constant volume to produce the solutions as $30.28 \mu \mathrm{g} / \mathrm{mL}, 31.14 \mu \mathrm{g} / \mathrm{mL}, 44.66 \mu \mathrm{g} / \mathrm{mL}$ and $27.31 \mu \mathrm{g} / \mathrm{mL}$, respectively.

Appropriate quantities of saikosaponin a and saikosaponin d were weighed accurately and dissolved in methanol, then constant volume to produce the solutions as $0.214 \mathrm{mg} / \mathrm{mL}$ and $0.197 \mathrm{mg} / \mathrm{mL}$, respectively.

\subsection{Optimization of Chromatographic Conditions}

In order to get optimal separation of complex components from Bupleuri Radix and Bupleuri Herba, six main factors containing the mobile phase composition, elution gradient, column temperature, detection wavelength, the best record time and extraction solvent were selected for optimization of chromatographic conditions.

\subsection{Validation of HPLC Fingerprint}

According to sample chromatographs of ten batches of Bupleuri Radix and Bupleuri Herba, respectively, it was found that Bupleuri Radix had 18 common fingerprint peaks (F1-F18) at 360nm and nine common peaks (S1-S9) at 203nm; and Bupleuri Herba also had 18 common fingerprint peaks (CF1-CF18) at 360nm and nine common peaks (CS1-CS9) at 203nm (sum of peaks area $>90 \%$ ). The common peak F5 / CF5 (rutin) and S5 / CS7 (saikosaponin d) of Bupleuri Radix and Bupleuri Herba were selected as reference peaks of flavonoids at $360 \mathrm{~nm}$ and saikosaponins at $203 \mathrm{~nm}$ for calculating relative retention time and relative peak area, respectively.

The proposed method for fingerprint analysis was validated in terms of precision, stability and repeatability. The validation was performed with sample XS1 of Bupleuri Radix and sample AH of Bupleuri Herba, respectively. The precision was assessed by analyzing six replicate injections, stability was tested at $0,2,4,8,12$, $24 \mathrm{~h}$ injections, and repeatability was performed with five replicate sample solutions by parallel preparation. The RSD values of relative retention time (RRT) and relative peak area (RPA) of peaks F1-F18 and S1-S9 in Bupleuri Radix and CF1-CF18 and CS1-CS9 in Bupleuri Herba were taken as a measure, respectively.

\subsection{Analysis of Chromatographic Fingerprints}

Similarity Evaluation for Chromatographic Fingerprint of Traditional Chinese Medicine was introduced to generate reference fingerprint developed with the mean or median of all chromatograms and to calculate the correlative coefficients between different chromatograms and the reference fingerprint [15]. In this study, peak F5 (rutin) or S5 (saikosaponin d) in Bupleuri Radix (HB3 sample) was assigned as the reference peak for flavonoids or saponins_to calculate RPA of each characteristic peak because they were the major common component peaks in the chromatogram of both Bupleuri Radix and Bupleuri Herba. The data were further processed using Microsoft Excel for averages and correlation coefficients (r) [16].

\section{Results and Discussion}

\subsection{Optimization of Chromatographic Conditions}

Four kinds of mobile phase system including acetonitrile-water, acetonitrile- $0.1 \%$ phosphoric acid water, 
acetonitrile- $0.1 \%$ formic acid water and methanol-water were investigated. The results showed that acetonitrile$0.1 \%$ phosphoric acid water gradient elution was best for good separation of both saikosaponins and flavonoids in $100 \mathrm{~min}$, the peaks shape were good, and the baseline is stable. The results also showed that $203 \mathrm{~nm}$ was suitable for detection saikosaponins and 360nm for flavonoids through comparison of 203nm, 254nm, 280nm, 360nm and $365 \mathrm{~nm}$ wavelengths. $35^{\circ} \mathrm{C}$ was better than $30^{\circ} \mathrm{C}$ and $40^{\circ} \mathrm{C}$ column temperature. The chromatograms of $120 \mathrm{~min}$ of sample solution and blank solvent showed that almost no component peaks were found after $95 \mathrm{~min}$, thus $100 \mathrm{~min}$ was the best record time. In addition, 70\% methanol was better than $50 \%$ and $100 \%$ methanol on considering of extraction efficiency of both saikosaponins and flavonoids.

\subsection{Validation of HPLC-DAD Fingerprint}

The precision results showed that the RSD values of RRTs for the common peaks F1-F18 and S1-S9 of Bupleuri Radix were $0.79 \% \sim 1.12 \%$ and $0.84 \% \sim 1.24 \%$, the RSD of RPAs were $1.49 \% \sim 3.72 \%$ and $1.40 \%$ $3.84 \%$, respectively; and the RSD of RRTs for the common peaks CF1-CF18 and CS1-CS9 of Bupleuri Herba were $0.20 \% \sim 1.11 \%$ and $0.15 \% \sim 1.41 \%$, the RSD of RPAs were $1.20 \% \sim 4.33 \%$ and $0.62 \% \sim 4.76 \%$, respectively.

The stability results showed that the RSD values of RRTs for Bupleuri Radix peaks F1-F18 and S1-S9 were $0.08 \% \sim 0.62 \%$ and $0.04 \% \sim 0.87 \%$, RSD of RPAs were $1.32 \% \sim 2.79 \%$ and $1.41 \% \sim 2.91 \%$, respectively; and the RSD of RRTs for Bupleuri Herba peaks CF1-CF18 and

CS1-CS9 were $0.06 \% \sim 0.41 \%$ and $0.11 \% \sim 0.68 \%$, RSD of RPAs were $0.76 \% \sim 2.83 \%$ and $0.66 \% \sim 2.79 \%$, respectively.

The repeatability results showed that the RSD values of RRTs for Bupleuri Radix peaks F1-F18 and S1-S9 were $0.07 \% \sim 1.01 \%$ and $0.04 \% \sim 0.76 \%$, RSD of RPAs were $0.34 \% \sim 4.26 \%$ and $0.95 \% \sim 4.43 \%$, respectively; and the RSD of RRTs for Bupleuri Herba peaks CF1-CF18 and CS1-CS9 were $0.02 \% \sim 0.88 \%$ and $0.05 \% \sim 0.53 \%$, RSD of RPAs were $0.18 \% \sim 4.16 \%$ and $0.47 \% \sim 4.33 \%$, respectively.

Above results indicated that the developed fingerprint for Bupleuri Radix and Bupleuri Herba are precise, stable and repeatable.

\subsection{Analysis of the Common Peaks}

Ten batches of Bupleuri Radix and Bupleuri Herba were analysed using the same samples preparation and chromatographic conditions (representative chromatograms were showed in Figure 1-A, B, C, D). The average of RRTs of 18 common fingerprint peaks (F1-F18 or CF1CF18) at 360nm and nine common peaks (S1-S9 or CS1CS9) at 203nm were regarded as the RRTs of the standard fingerprint chromatogram of Bupleuri Radix or Bupleuri Herba, repectively, and the RRTs of sample chromatograms should be in the range of mean $\pm 10 \%$. The results showed that the RSD of RRTs of common peaks of both Bupleuri Radix and Bupleuri Herba were less than 3\%, which completely corresponded with the relevant requirements of "fingerprint of traditional Chinese medicine injection technology requirements" issued by the State. Therefore, chromatograms composed with above common peaks can be taken as HPLC-DAD chromatographic fingerprint of Bupleuri Radix or Bupleuri Herba.

\subsection{Fingerprint Analysis and Comparison of Bupleuri Radix and Bupleuri Herba}

It can be seen from the fingerprint chromatograms that 18 major flavonoids were detected in both Bupleuri Radix and Bupleuri Herba, respectively, and 12 component peaks among which (F1/CF1, F2/CF2, F4/CF4, F5/CF5, F6/CF6, F7/CF7, F8/CF10, F10/CF11, F12/CF12, F13/CF13, F14/CF14, F15/CF17) were shared by these two herb medicines; six component peaks (F3, F9, F11, F16, F17 and F18 or CF3, CF8, CF9, CF15, CF16 and CF18) were specific for Bupleuri Radix or Bupleuri Herba, respectively. By comparison with reference substance, peaks F5/CF5, F12/CF12, F13/CF13, F14/CF14 were identified as rutin, quercetin, kaempferide and isorhamnetin, respectively.

Nine major saponins were detected in both Bupleuri Radix and Bupleuri Herba, including four common ingredients peaks (S3/CS2, S4/CS3, S5/CS7, S9/CS9) for Bupleuri Radix and Bupleuri Herba; five component peaks (S1, S2, S6, S7 and S8 or CS1, CS4, CS5, CS6 and CS8) were specific for Bupleuri Radix or Bupleuri Herba, respectively. Resorting to reference substances, peaks S3/CS2 and S5/CS7 were identified as saikosaponin a and saikosaponin d, respectively.

Peak F5/CF5 (rutin) or S5/CS7 (saikosaponin d) was assigned as the reference peak (RP) for flavonoids or saponins, because they were the major common component peaks in the chromatogram of both Bupleuri Radix and Bupleuri Herba. The areas of all other peaks (flavonoids or saponins) in the chromatogram are normalized against the area of the RP in Bupleuri Radix (HB3 sample) (expressed as the ratios to the RP) (Table 1 and Table 2), and the standard fingerprint of Bupleuri Radix or Bupleuri Herba was got from the average of relative area of fingerprint peaks, respectively. Correlation analysis of ten batches of Bupleuri Radix or ten batches of Bupleuri Herba fingerprint was carried out by calculating correlation coefficient (r) compared with their standard fingerprint, respectively. The results were showed in Table 1 and Table 2. Correlation coefficient of flavonoids of ten batches of Bupleuri Radix can be divided into two groups: five batches showed good correlation $(\mathrm{r}=0.88 \sim$ 0.99), while the other five batches with poor correlation ( $\mathrm{r}$ $=-0.09 \sim 0.38$ ); while the correlation coefficient of saponins varied less than that of flavonoids, they were above 0.6 except sample HB4 with $r=0.49$. As for ten batches of Bupleuri Herba, the correlation coefficient of both flavonoids and saponins can also be divided into two groups: seven batches of good correlation $(\mathrm{r}=0.88 \sim 0.98)$ and three batches of poor correlation $(r=0.34 \sim 0.35)$ for flavonoids; seven batches of good correlation $(r=0.95 \sim$ 0.99 except one batch of 0.58 ) and three batches of poor correlation $(r=0.01 \sim 0.05)$ for saponins. Correlation coefficients of flavonoids and saponins of standard fingerprint of Bupleuri Radix and Bupleuri Herba were 0.04 and -0.18 , respectively. In addition, the sum of the average relative peak area of flavonoids and saponins of fingerprint peaks of ten batch of Bupleuri Herba was 9 and 1.4 times to that of Bupleuri Radix, respectively. Above results indicated that the material basis (especially 
flavonoids) of Bupleuri Radix and Bupleuri Herba varied significantly.
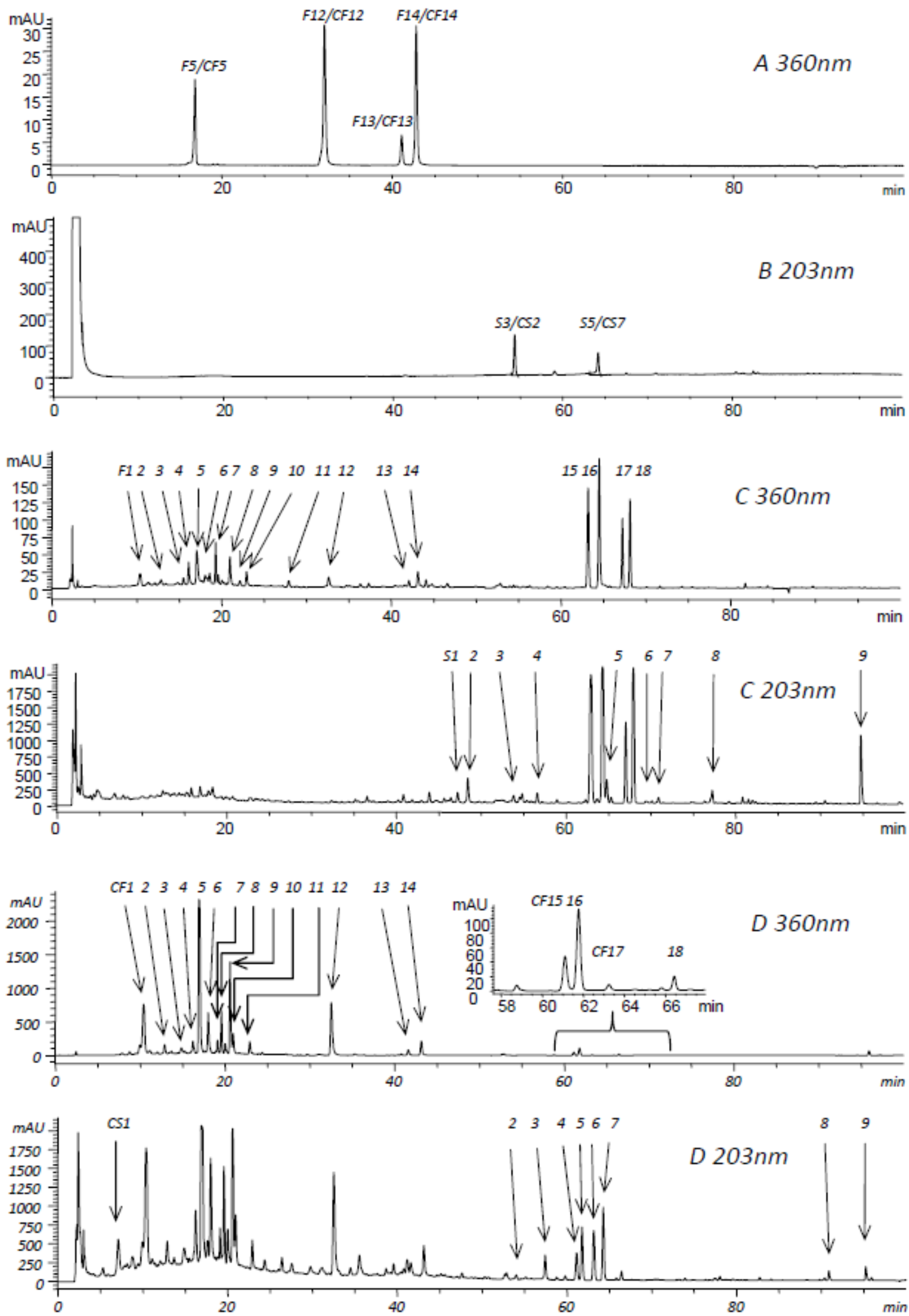

Figure 1. HPLC chromatograms of reference substances (A, B), Bupleuri Radix (C) and Bupleuri Herba (D). F5/CF5: Rutin, F12/CF12: quercetin, F13/CF13: kaempferide, F14/CF14: isorhamnetin, S3/CS2: saikosaponin a, S5/CS7: saikosaponin d 
Table 1. Comparative analysis of chromatographic fingerprint of flavonoid components between Bupleuri Radix and Bupleuri Herba

\begin{tabular}{|c|c|c|c|c|c|c|c|c|c|c|c|c|}
\hline \multirow{2}{*}{$\begin{array}{l}\text { Peaks } \\
\text { Bupleuri } \\
\text { Radix } \\
\end{array}$} & \multirow[t]{2}{*}{ Retention time tR(min) } & \multicolumn{10}{|c|}{ Relative peak area } & \multirow[t]{2}{*}{ mean } \\
\hline & & HB1 & HB2 & $\mathrm{AH}$ & SX1 & SX2 & NM & HB3 & HB4 & SX3 & SX4 & \\
\hline F1/CF1 & 10.34 & 0.02 & 0.02 & 0.06 & 0.02 & 0.01 & 0.39 & 0.18 & 0.09 & 0.15 & 0.04 & 0.10 \\
\hline F2/CF2 & 12.87 & 0.00 & 0.00 & 0.06 & 0.06 & 0.06 & 0.09 & 0.09 & 0.06 & 0.08 & 0.06 & 0.06 \\
\hline CF3 & 14.78 & 0 & 0 & 0 & 0 & 0 & 0 & 0 & 0 & 0 & 0 & 0 \\
\hline F3 & 15.71 & 0.00 & 0.00 & 0.03 & 0.01 & 0.01 & 0.00 & 0.22 & 0.15 & 0.07 & 0.09 & 0.06 \\
\hline F4/CF4 & 16.09 & 0.00 & 0.01 & 0.01 & 0.00 & 0.01 & 0.05 & 0.71 & 0.51 & 0.21 & 0.26 & 0.18 \\
\hline F5/CF5 & 17.01 & 0.04 & 0.03 & 0.15 & 0.19 & 0.17 & 0.47 & 1.00 & 0.65 & 0.44 & 0.45 & 0.36 \\
\hline F6/CF6 & 18.03 & 0.09 & 0.11 & 0.05 & 0.04 & 0.11 & 0.16 & 0.22 & 0.15 & 0.13 & 0.10 & 0.12 \\
\hline F7/CF7 & 19.17 & 0.01 & 0.03 & 0.16 & 0.83 & 1.08 & 0.14 & 0.04 & 0.01 & 0.29 & 0.40 & 0.30 \\
\hline CF8 & 19.64 & 0 & 0 & 0 & 0 & 0 & 0 & 0 & 0 & 0 & 0 & 0 \\
\hline CF9 & 20.10 & 0 & 0 & 0 & 0 & 0 & 0 & 0 & 0 & 0 & 0 & 0 \\
\hline F8/CF10 & 21.06 & 0.05 & 0.04 & 0.29 & 0.12 & 0.10 & 0.55 & 0.18 & 0.12 & 0.27 & 0.20 & 0.19 \\
\hline F9 & 22.93 & 0.01 & 0.01 & 0.07 & 0.05 & 0.04 & 0.09 & 0.06 & 0.03 & 0.05 & 0.06 & 0.05 \\
\hline F10/CF11 & 23.03 & 0.03 & 0.03 & 0.09 & 0.08 & 0.06 & 0.26 & 0.11 & 0.06 & 0.13 & 0.11 & 0.10 \\
\hline F11 & 28.84 & 0.02 & 0.28 & 0.01 & 0.00 & 0.02 & 0.00 & 0.00 & 0.17 & 0.05 & 0.00 & 0.06 \\
\hline F12/CF12 & 32.75 & 0.04 & 0.02 & 0.04 & 0.07 & 0.06 & 0.17 & 0.12 & 0.16 & 0.12 & 0.08 & 0.09 \\
\hline F13/CF13 & 41.80 & 0.04 & 0.02 & 0.01 & 0.06 & 0.06 & 0.14 & 0.03 & 0.04 & 0.05 & 0.03 & 0.05 \\
\hline F14/CF14 & 43.30 & 0.11 & 0.04 & 0.07 & 0.08 & 0.08 & 0.29 & 0.09 & 0.09 & 0.17 & 0.08 & 0.11 \\
\hline CF15 & 61.12 & 0 & 0 & 0 & 0 & 0 & 0 & 0 & 0 & 0 & 0 & 0 \\
\hline CF16 & 61.78 & 0 & 0 & 0 & 0 & 0 & 0 & 0 & 0 & 0 & 0 & 0 \\
\hline F15/CF17 & 63.26 & 0.00 & 0.01 & 0.00 & 0.00 & 0.01 & 1.85 & 1.22 & 1.00 & 0.96 & 0.42 & 0.55 \\
\hline F16 & 64.11 & 0.00 & 0.01 & 0.02 & 0.35 & 0.45 & 1.78 & 2.01 & 1.54 & 1.20 & 0.84 & 0.82 \\
\hline CF18 & 66.44 & 0.00 & 0.00 & 0.00 & 0.00 & 0.00 & 0.00 & 0.00 & 0.00 & 0.00 & 0.00 & 0.00 \\
\hline F17 & 67.24 & 0.00 & 0.00 & 0.00 & 0.00 & 0.01 & 0.48 & 1.38 & 1.12 & 0.54 & 0.48 & 0.40 \\
\hline \multirow[t]{2}{*}{ F18 } & 68.15 & 0.01 & 0.00 & 0.00 & 0.00 & 0.01 & 0.59 & 1.84 & 1.41 & 0.68 & 0.64 & 0.52 \\
\hline & Correlation coefficient $\mathrm{r}$ & -0.09 & -0.10 & 0.08 & 0.38 & 0.38 & 0.88 & 0.93 & 0.93 & 0.99 & 0.97 & $\begin{array}{c}\text { Mean together } \\
4.1\end{array}$ \\
\hline $\begin{array}{l}\text { Bupleuri } \\
\text { Herba } \\
\end{array}$ & & $\mathrm{AH}$ & HB1 & HB2 & NM1 & HB3 & HB4 & HB5 & QJ1 & QJ2 & QJ3 & \\
\hline F1/CF1 & 10.34 & 7.69 & 9.11 & 5.55 & 2.11 & 7.48 & 6.05 & 3.81 & 0.94 & 0.85 & 1.12 & 4.47 \\
\hline F2/CF2 & 12.87 & 1.29 & 0.95 & 0.72 & 1.11 & 0.57 & 1.49 & 0.00 & 0.66 & 0.69 & 0.68 & 0.82 \\
\hline CF3 & 14.78 & 0.71 & 1.33 & 0.61 & 0.37 & 0.61 & 1.07 & 0.00 & 0.00 & 0.00 & 0.00 & 0.47 \\
\hline F3 & 15.71 & 0 & 0 & 0 & 0 & 0 & 0 & 0 & 0 & 0 & 0 & 0.0 \\
\hline F4/CF4 & 16.09 & 1.12 & 0.57 & 0.56 & 0.36 & 0.52 & 4.37 & 0.00 & 0.00 & 0.00 & 0.00 & 0.75 \\
\hline F5/CF5 & 17.01 & 20.11 & 23.29 & 16.55 & 5.57 & 20.19 & 13.68 & 16.00 & 0.36 & 0.29 & 0.47 & 11.65 \\
\hline F6/CF6 & 18.03 & 4.52 & 3.63 & 3.08 & 0.54 & 2.34 & 7.29 & 0.94 & 8.34 & 9.84 & 9.37 & 4.99 \\
\hline F7/CF7 & 19.17 & 1.19 & 1.31 & 0.85 & 0.19 & 1.11 & 1.62 & 0.43 & 0.20 & 0.21 & 0.02 & 0.71 \\
\hline CF8 & 19.64 & 3.45 & 4.00 & 2.87 & 0.64 & 2.86 & 3.06 & 2.05 & 0.32 & 0.42 & 0.28 & 2.00 \\
\hline CF9 & 20.10 & 1.31 & 0.71 & 1.78 & 0.33 & 0.60 & 1.55 & 1.62 & 0.21 & 0.14 & 0.16 & 0.84 \\
\hline F8/CF10 & 21.06 & 2.18 & 2.64 & 1.13 & 0.40 & 2.22 & 1.56 & 1.64 & 1.36 & 1.63 & 1.94 & 1.67 \\
\hline F9 & 22.93 & 0 & 0 & 0 & 0 & 0 & 0 & 0 & 0 & 0 & 0 & 0 \\
\hline F10/CF11 & 23.03 & 1.68 & 1.97 & 0.63 & 0.22 & 2.07 & 0.84 & 1.60 & 0.52 & 0.51 & 0.56 & 1.06 \\
\hline F11 & 28.84 & 0 & 0 & 0 & 0 & 0 & 0 & 0 & 0 & 0 & 0 & 0 \\
\hline F12/CF12 & 32.75 & 6.89 & 4.10 & 8.54 & 4.93 & 10.28 & 5.80 & 6.89 & 0.22 & 0.64 & 0.26 & 4.85 \\
\hline F13/CF13 & 41.80 & 0.60 & 0.27 & 0.34 & 0.17 & 0.44 & 0.73 & 0.16 & 0.10 & 0.09 & 0.14 & 0.30 \\
\hline F14/CF14 & 43.30 & 1.49 & 0.67 & 1.73 & 0.87 & 1.37 & 1.42 & 1.08 & 0.08 & 0.19 & 0.07 & 0.90 \\
\hline CF15 & 61.12 & 0.33 & 0.16 & 0.44 & 0.29 & 0.16 & 0.17 & 0.00 & 0.00 & 0.00 & 0.00 & 0.15 \\
\hline CF16 & 61.78 & 0.76 & 0.45 & 1.36 & 0.46 & 0.43 & 0.30 & 0.00 & 0.00 & 0.00 & 0.00 & 0.38 \\
\hline F15/CF17 & 63.26 & 0.05 & 0.05 & 0.00 & 0.00 & 0.05 & 0.02 & 0.00 & 0.00 & 0.00 & 0.00 & 0.02 \\
\hline F16 & 64.11 & 0 & 0 & 0 & 0 & 0 & 0 & 0 & 0 & 0 & 0 & 0 \\
\hline CF18 & 66.44 & 0.11 & 0.12 & 0.59 & 0.14 & 0.28 & 0.15 & 0.00 & 0.00 & 0.00 & 0.00 & 0.14 \\
\hline F17 & 67.24 & 0 & 0 & 0 & 0 & 0 & 0 & 0 & 0 & 0 & 0 & 0 \\
\hline \multirow[t]{2}{*}{ F18 } & 68.15 & 0 & 0 & 0 & 0 & 0 & 0 & 0 & 0 & 0 & 0 & 0 \\
\hline & Correlation coefficient $\mathrm{r}$ & 0.98 & 0.95 & 0.96 & 0.88 & 0.96 & 0.97 & 0.94 & 0.34 & 0.34 & 0.35 & $\begin{array}{c}\text { Mean } \\
\text { together :36.2 }\end{array}$ \\
\hline
\end{tabular}


Table 2. Comparative analysis of chromatographic fingerprint of saponin components between Bupleuri Radix and Bupleuri Herba

\begin{tabular}{|c|c|c|c|c|c|c|c|c|c|c|c|c|}
\hline \multirow{2}{*}{$\begin{array}{l}\text { peaks } \\
\text { Bupleuri Radix }\end{array}$} & \multirow[t]{2}{*}{ Retention time tR(min) } & \multicolumn{10}{|c|}{ Relative peak area } & \multirow[t]{2}{*}{ mean } \\
\hline & & HB1 & HB2 & $\mathrm{AH}$ & SX1 & SX2 & NM & HB3 & HB4 & SX3 & SX4 & \\
\hline CS1 & 7.02 & 0 & 0 & 0 & 0 & 0 & 0 & 0 & 0 & 0 & 0 & 0 \\
\hline S1 & 47.29 & 0.39 & 0.29 & 0.47 & 0.67 & 0.23 & 0.79 & 0.68 & 0.72 & 0.90 & 0.55 & 0.57 \\
\hline S2 & 48.28 & 0.02 & 0.02 & 0.09 & 0.01 & 0.08 & 2.56 & 1.85 & 2.12 & 1.61 & 0.66 & 0.90 \\
\hline S3/CS2 & 54.11 & 1.80 & 1.74 & 2.11 & 0.44 & 0.45 & 0.86 & 0.46 & 0.45 & 0.54 & 1.07 & 0.99 \\
\hline CS4 & 61.03 & 0 & 0 & 0 & 0 & 0 & 0 & 0 & 0 & 0 & 0 & 0 \\
\hline CS5 & 61.72 & 0 & 0 & 0 & 0 & 0 & 0 & 0 & 0 & 0 & 0 & 0 \\
\hline CS6 & 63.14 & 0 & 0 & 0 & 0 & 0 & 0 & 0 & 0 & 0 & 0 & 0 \\
\hline S5/CS7 & 64.21 & 1.04 & 1.21 & 1.38 & 1.67 & 1.66 & 1.00 & 1.00 & 0.95 & 1.18 & 0.81 & 1.19 \\
\hline S6 & 69.73 & 0.02 & 0.06 & 0.00 & 1.31 & 1.31 & 0.28 & 0.03 & 0.06 & 0.59 & 0.50 & 0.42 \\
\hline S7 & 71.12 & 0.01 & 0.12 & 0.45 & 7.76 & 7.57 & 0.00 & 0.46 & 0.27 & 2.93 & 3.03 & 2.26 \\
\hline S8 & 77.93 & 0.05 & 0.06 & 0.25 & 0.05 & 0.14 & 0 & 1.02 & 2.26 & 1.11 & 0.50 & 0.54 \\
\hline CS8 & 90.79 & 0 & 0 & 0 & 0 & 0 & 0 & 0 & 0 & 0 & 0 & 0 \\
\hline \multirow[t]{2}{*}{ S9/CS9 } & 95.17 & 2.20 & 2.32 & 2.76 & 1.27 & 1.05 & 3.14 & 4.31 & 2.05 & 2.22 & 2.81 & 2.41 \\
\hline & Correlation coefficient $\mathrm{r}$ & 0.62 & 0.66 & 0.71 & 0.70 & 0.68 & 0.61 & 0.72 & 0.49 & 0.93 & 0.98 & Mean together : 9.6 \\
\hline Bupleuri Herba & & $\mathrm{AH}$ & HB1 & HB2 & NM1 & HB3 & HB4 & HB5 & QJ1 & QJ2 & QJ3 & \\
\hline CS1 & 7.02 & 2.85 & 1.61 & 1.70 & 1.28 & 1.78 & 1.56 & 4.08 & 0.29 & 0.29 & 0.35 & 1.58 \\
\hline S1 & 47.29 & 0 & 0 & 0 & 0 & 0 & 0 & 0 & 0 & 0 & 0 & 0 \\
\hline S2 & 48.28 & 0 & 0 & 0 & 0 & 0 & 0 & 0 & 0 & 0 & 0 & 0 \\
\hline S3/CS2 & 54.11 & 0.18 & 0.13 & 0.21 & 0.14 & 0.13 & 0.14 & 0.17 & 0.01 & 0.02 & 0.04 & 0.12 \\
\hline S4/CS3 & 57.00 & 2.21 & 2.32 & 4.18 & 1.54 & 2.31 & 1.98 & 0.50 & 0.10 & 0.05 & 0.06 & 1.52 \\
\hline CS4 & 61.03 & 2.40 & 1.17 & 2.51 & 1.42 & 1.26 & 2.11 & 0.14 & 0.05 & 0.02 & 0.01 & 1.11 \\
\hline CS5 & 61.72 & 3.75 & 2.30 & 6.18 & 2.18 & 2.20 & 1.72 & 0.07 & 0.01 & 0.03 & 0.04 & 1.85 \\
\hline S6 & 69.73 & 0 & 0 & 0 & 0 & 0 & 0 & 0 & 0 & 0 & 0 & 0 \\
\hline S7 & 71.12 & 0 & 0 & 0 & 0 & 0 & 0 & 0 & 0 & 0 & 0 & 0 \\
\hline S8 & 77.93 & 0 & 0 & 0 & 0 & 0 & 0 & 0 & 0 & 0 & 0 & 0 \\
\hline CS8 & 90.79 & 0.52 & 0.22 & 0.02 & 0.63 & 0.43 & 0.48 & 0.84 & 0.57 & 0.63 & 0.43 & 0.48 \\
\hline \multirow[t]{2}{*}{ S9/CS9 } & 95.17 & 0.74 & 0.42 & 0.51 & 1.06 & 0.82 & 0.70 & 1.52 & 1.32 & 1.26 & 0.87 & 0.92 \\
\hline & Correlation coefficient $\mathrm{r}$ & 0.97 & 0.97 & 0.95 & 0.99 & 0.98 & 0.97 & 0.58 & 0.05 & 0.01 & 0.03 & Mean together : 13.8 \\
\hline
\end{tabular}

HB presents for Hebei, AH for Anhui, SX for Shanxi, NM for Neimeng, QJ for Quanjiao in Anhui.

\section{Conclusion}

The present optimal chromatographic conditions combined with diode array detection (DAD) had realized effective separation and stimutanous detection of flavonoids and saponins of both Bupleuri Radix and Bupleuri Herb in 100 mins. 24 major flavonoids peaks were detected from the newly developed HPLC-DAD fingerprints, among which 12 peaks were shared by these two herbs, and six peaks were special for Bupleuri Radix or Bupleuri Herb, respectively. Meanwhile, 14 major saponin peaks were detected, and of which only four peaks were shared by the two herbs, while five peaks were special for each herb. Correlation analysis showed that Bupleuri Radix or Bupleuri Herb can be divided into two groups, and the general correlation between Bupleuri Radix and Bupleuri Herb was poor. The current HPLCDAD fingerprints can reflect the chemical composition and their differences of Bupleuri Radix and Bupleuri Herb in a rational and effective way. Comparative analysis of chromatographic fingerprint indicated that the material basis of Bupleuri Radix and Bupleuri Herb varied significantly, which hinted that clinical application of
Bupleuri Herb instead of Bupleuri Radix should be carefully considered.

\section{Acknowledgements}

This work was supported by Natural Science Foundation of Jiangsu Province of China (BK20141507, BK2011866), and also in part by Foundation for HighLevel Talent in Six Areas of Jiangsu Province (No. 2013YY006).

\section{References}

[1] Chinese Pharmacopoeia Commission, Chinese Pharmacopoeia of the People's Republic of China (Vol. 1), Bejing: China Medical Science and Technology Press, p. 263, 2010.

[2] Huang H.Q., Zhang X., Xu Z.X., Su J., Yan S.K., Zhang W.D., "Fast determination of saikosaponins in Bupleurum by rapid resolution liquid chromatography with evaporative light scattering detection”, Journal of Pharmaceutical and Biomedical Analysis, 49(4), 1048-1055, 2009.

[3] Yang Z.Y., Liu S.F., Zhao Z., Pan S.L., "Determination of saikosaponin a, c, d of 4 species of Radix Bupleuri in Xinjiang by HPLC”, China Journal of Chinese Materia Medica, 33(4), 460461, 2008. 
[4] Lin D.H., Mao R.G., Wang Z.H., Hong X.K., Pan S.L., "Quantitative analysis of saikosaponin a, c, d from 23 species of Radix Bupleuri in China by RP-HPLC”, Chinese Journal of Pharmaceutical Analysis, 24(5), 479-483, 2004.

[5] Zhou Y., Wang Y.L., Ma Z.Q., Wang B., Liang H., Zhao Y.Y., Zhang Q.Y., "Chromatographic fingerprint analysis of Bupleuri Radix by HPLC-ELSD”, Journal of Chinese Pharmaceutical Sciences, 22(1), 40-46, 2013.

[6] Li J., Wang B., Zhang L., Pan L.L., Zhao M., OuYang Z., "Study of the content of flavonoids and HPLC fingerprint in Bupleurum scorzonerifolium Willd.”, Journal of Jiangsu University (Medicine Edition), 19(2), 139-142, 2009.

[7] Wang B., OuYang Z., Zhao M., Pan L.L., Zhang L., "Study on HPLC characteristic chromatograms on aerial parts of Bupleurum scorzonerifolium and qualitative analysis by HPLC-ESI-TOFMS”, Chinese Journal of Pharmaceutical Analysis, 29(7), 1099-1103, 2009.

[8] Tan L.L., Cai X., Hu Z.H., "Dynamic changes of total flavonoids of Bupleurum chinense DC”, Chinese Traditional and Herbal Drugs, 39(2), 286-287, 2008.

[9] Zhang T.T., Zhou J.S., Wang Q., "HPLC analysis of flavonoids from the aerial parts of Bupleurum species", Chinese Journal of Natural Medicines, 8(2), 0107-0113, 2010.

[10] Zhou T.H., "Determination of saikosaponin a,d of Bupleuri Radix and Bupleuri Herba by HPLC”, China Pharmacist, 13(2), 293-294, 2010.
[11] Wei Y.J., Wang M., Ning Q., Zhan W., Shi J.Y., Jia X.B., "HPLC simultaneous determination of saponins and flavonoids of Bupleuri Radix and Bupleuri Herba”, Chinese Journal of Pharmaceutical Analysis, 31(5), 879-883, 2011.

[12] Wu W.Y., Guo D.A., "Strategies for elaboration of comprehensive quality standard system on traditional Chinese medicine”, China Journal of Chinese Materia Medica, 39(3), 351-356, 2014.

[13] Tang H.M., Luo Y.M., Yan Y., Liang Q.L., Meng X.S., Luo G.A., "Research method of traditional Chinese medicine fingerprint based on diode-array detector", Chemical Journal of Chinese Universities, 30(2), 279-282, 2009.

[14] Xie P.S., Yan Y.Z., Guo B.L., Lam C.W., Chui S.H., Yu Q.X., "Chemical pattern-aided classification to simplify the intricacy of morphological taxonomy of Epimedium species using chromatographic fingerprinting”, Journal of Pharmaceutical and Biomedical Analysis, 52(4), 452-460, 2010.

[15] Wu Q.Y., Zhou Y., Jin X., Guan Y., Xu M., Liu L.F., "Chromatographic fingerprint and the simultaneous determination of five bioactive components of Geranium carolinianum L. water extract by high performance liquid chromatography", International Journal of Molecular Sciences, 12(12), 8740-8749, 2011.

[16] Wei J.R., Ma Y.H., "The similarity calculation of chromatography fingerprint with Microsoft Excel software”, Journal of Kunming Teachers College, 29(4), 110-112, 2007. 